\title{
Comparison of the original and the spectral system of an elastic rotor in two-lobe bearings with an active geometry variation
}

\author{
Kai Becker ${ }^{1, *}$ and Wolfgang Seemann ${ }^{1}$ \\ ${ }^{1}$ Karlsruhe Institute of Technology, Institute of Engineering Mechanics
}

\begin{abstract}
The improvement of the dynamic behaviour of an elastic rotor which is supported by journal bearings represents an ongoing field of research, whereby various modifications of the corresponding bearings and their geometry have been proposed in literature. In order to suppress or at least to decrease unwanted oscillations due to negative effects of instability phenomena of the 'oil-whirl'- and/or 'oil-whip'-type, a two-lobe bearing with an active geometry variation is suggested as an alternative approach. A systematic investigation of the dynamic behaviour of a Jeffcott rotor, which is supported by the mentioned bearing type, is performed by means of the associated spectral system.
\end{abstract}

\section{Modelling}

\subsection{Pressure and Bearing Forces}

The non-dimensional pressure $\Pi$ in the two-lobe bearing is modelled according to the non-dimensional Reynolds equation under consideration of the short-bearing approach (cf. [1]):

$$
\gamma^{2} \frac{\partial}{\partial \bar{z}}\left(\frac{\partial \Pi}{\partial \bar{z}} H^{3}\right)=6 \frac{\partial H}{\partial \varphi}+12 \frac{\partial H}{\partial \tau} \quad \text { with } \quad H=1 \underbrace{\widehat{\widehat{D}\left(1+\delta_{D} \cos (\Omega \tau)\right)|\sin \varphi|}}_{\text {= harmonic geometry variation }}-X_{B} \cos \varphi-Y_{B} \sin \varphi
$$

The fluid-film-thickness $H$ depends on the time-dependent geometry variation (controlled by $\widehat{D}, \delta_{D}, \Omega$ ), which corresponds to a simultaneous back-and-forth motion of the upper and lower lobe. The non-dimensional coordinates of the journal are given by $\left(X_{B}, Y_{B}\right)$. The bearing forces $f_{x}$ and $f_{y}$ are obtained via integration of the positive pressure values $\max (\Pi, 0)$ over the journal's surface. A detailed description of the bearing and the corresponding pressure modelling can be found in [2].

\subsection{Jeffcott Rotor}

With the determined bearing forces $f_{x}$ and $f_{y}$ the equations of motion of a perfectly balanced Jeffcott rotor with nondimensional rotor coordinates $\left(X_{B}, Y_{B}\right)$ can be obtained (time derivative denoted by: $\left.(. .)^{\prime}=\mathrm{d} / \mathrm{d} \tau\right)$ :

$$
\begin{aligned}
\bar{\omega}^{2} X_{R}^{\prime \prime}+\bar{d}_{a} \bar{\omega} X_{R}^{\prime}+\frac{X_{R}-X_{B}}{\Gamma} & =f, & \eta \bar{\omega}^{2} X_{B}^{\prime \prime}+\frac{X_{B}-X_{R}}{\Gamma}-2 \sigma \bar{\omega} f_{x} & =0, \\
\bar{\omega}^{2} Y_{R}^{\prime \prime}+\bar{d}_{a} \bar{\omega} Y_{R}^{\prime}+\frac{Y_{R}-Y_{B}}{\Gamma} & =0, & \eta \bar{\omega}^{2} Y_{B}^{\prime \prime}+\frac{Y_{B}-Y_{R}}{\Gamma}-2 \sigma \bar{\omega} f_{y} & =0 .
\end{aligned}
$$

A detailed description of the used parameters can be found as well in [2]. For the following analysis the most relevant parameters are given by the rpm parameter $\bar{\omega}$ and the bearing variation parameters $\widehat{D}, \delta_{D}$ and $\Omega$.

\subsection{Spectral System}

As the time-varying bearing geometry enters the equations of motion (2) as a parameter, the system is exposed to parametric as well as self-excitation, which can lead to quasi-periodic behaviour. Therefore, the associated spectral system is derived according to the suggested method of Schilder et al. [3]. Like described in [4] the equations of motion are transformed to a first-order system and an invariance equation for the torus function $u\left(\theta_{1}, \theta_{2}\right): \mathbb{T}^{2} \rightarrow \mathbb{R}^{8}$ is set up. The invariance equation is approximately solved by means of a Galerkin approach, whereby only the first harmonics are chosen as base functions:

$$
\underset{\sim}{u} \approx \sim_{\sim}^{*}=\sum_{k=1}^{3}{\underset{\sim}{u}}_{k}\left(\theta_{2}\right) \varphi_{k}\left(\theta_{1}\right) \quad \text { with } \quad\left[\varphi_{1}, \varphi_{2}, \varphi_{3}\right]^{T}=\left[1, \sin \left(\theta_{1}\right), \cos \left(\theta_{1}\right)\right]^{T}, \quad{\underset{\sim}{u}}_{k}\left(\theta_{2}\right): \mathbb{T}^{1} \rightarrow \mathbb{R}^{8} .
$$

Setting up the residual and performing the Galerkin projections leads to a system of $8 \times 3=24$ ordinary differential equations in $\theta_{2}$, the spectral system. It is used to approximate the torus function $u\left(\theta_{1}, \theta_{2}\right)$ and by association also the torus-manifold on which the stationary system trajectories might be located. An equilibrium solution of the spectral system can thereby correspond to periodic behaviour of the original system, whereas a periodic solution can correspond to quasi-periodic behaviour.

\footnotetext{
* Corresponding author: e-mail kai.becker@kit.edu, phone +49721608 46823, fax +4972160846070
} 


\section{Results}

The spectral system is investigated for its stationary solutions and compared to simulation results of the original system. Furthermore, an equilibrium continuation of the spectral system depending on the rpm parameter $\bar{\omega}$ is performed.
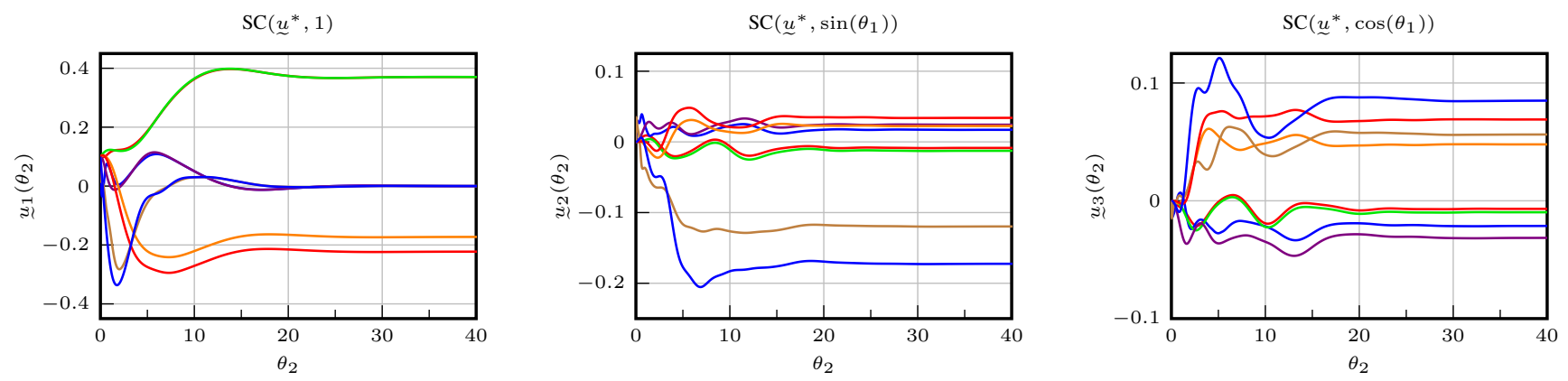

Fig. 1: Spectral Components (SC) of $\underline{\sim}^{*}\left(\theta_{1}, \theta_{2}\right)$ for $\sigma=0.05, \eta=0.1, \Gamma=0.1, \bar{d}_{a}=1, f=1, \bar{\omega}=2, \widehat{D}=0.2, \delta_{D}=0.3, \Omega=2.5$.

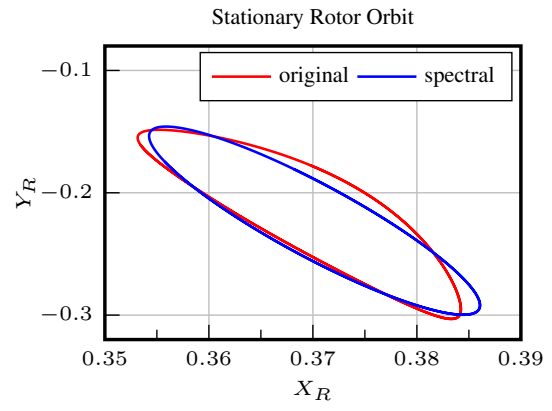

Fig. 2: Comparison of stationary rotor trajectory for parameter-set from Fig. 1
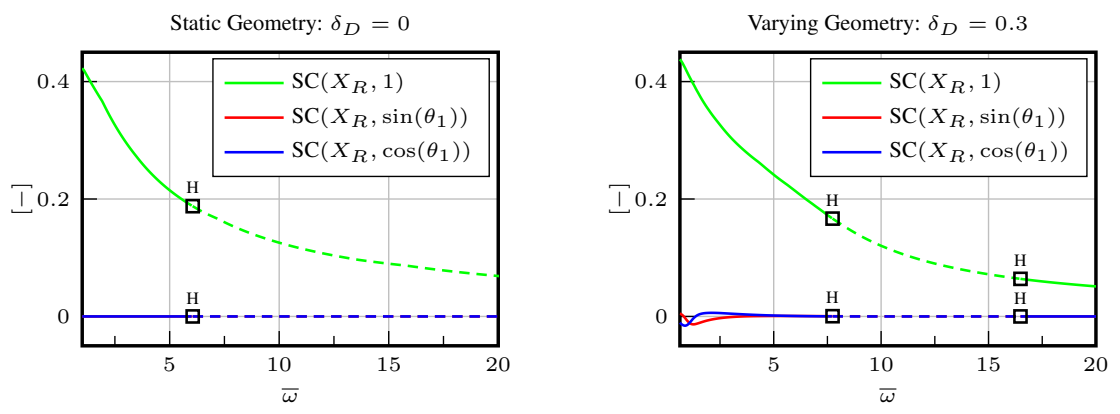

Fig. 3: Equilibria of the spectral system, i.e. the spectral components of the coordinate $X_{R}$, in dependence of $\bar{\omega}$ for $\delta_{D}=0$ (left) and $\delta_{D}=0.3$ (right) for parameter-set from Fig. 1

Some exemplary integration results of the spectral system are given in Fig. 1, i.e. the different spectral components (SC) associated with the corresponding base functions acc. to approach (3) are depicted. The different colours represent the individual entries of the corresponding elements $\underset{\sim}{u}(k=1,2,3)$. The detected equilibrium solution of the spectral system is used to construct an approximation of the stationary trajectory, which is compared to the corresponding simulation result of the original system (cf. Fig. 2). Although just the first harmonics have been chosen as base functions, the approximation via the spectral system leads to qualitatively accurate results. In contrast to classical approaches of finding periodic solutions the depicted approximation has been found through an equilibrium search of the spectral system.

In Fig. 3 an equilibrium continuation of the spectral system in dependence of the rpm parameter $\bar{\omega}$ is shown. It is distinguished between the two configurations without $\left(\delta_{D}=0\right)$ and with geometry variation $\left(\delta_{D} \neq 0\right)$. In the case of a static geometry the equilibrium solution loses its stability via a Hopf bifurcation $(\mathrm{H})$ at $\bar{\omega} \approx 6$ and stays instable for the remaining parameter range. In contrast, the equilibrium solution of the system with geometry variation has a larger stable range at low values of $\bar{\omega}$ and in addition it even regains its stability with increasing rpm parameter at $\bar{\omega} \approx 16$.

\section{Conclusion}

It has been shown that the periodic behaviour of the parametrically excited rotor system can be well approximated by means of equilibria of the associated spectral system. Furthermore, a stabilizing effect of the geometry variation on the Jeffcot rotor system has been detected via an equilibrium continuation of the spectral system, which of course highly depends on the chosen values of $\widehat{D}, \delta_{D}$ and $\Omega$.

\section{References}

[1] A. Z. Szeri, "Fluid film lubrication", Cambridge University Press (2005).

[2] K. Becker and W. Seemann, "A Journal Bearing with actively modified geometry for extending the parameter-based stability range of rotor dynamic systems", International Symposium on Transport Phenomena and Dynamics of Rotating Machinery (2016).

[3] F. Schilder, W. Vogt, S. Schreiber, and H.M. Osinga, "Fourier methods for quasi-periodic oscillations", International journal for numerical methods in engineering, 67(5), 2006.

[4] Becker, K. and Seemann, W. (2016), "Approximation of quasi-periodic solutions of a rotor in two-lobe bearings with time-varying geometry”, Proc. Appl. Math. Mech., 16: 263-264. doi:10.1002/pamm.201610120 


\section{Repository KITopen}

Dies ist ein Postprint/begutachtetes Manuskript.

Empfohlene Zitierung:

Becker, K.; Seemann, W.

Comparison of the original and the spectral system of an elastic rotor in two-lobe bearings with an active geometry variation.

2017. Proceedings in applied mathematics and mechanics, 17.

doi: $10.5445 / / R / 1000090324$

Zitierung der Originalveröffentlichung:

Becker, K.; Seemann, W.

Comparison of the original and the spectral system of an elastic rotor in two-lobe bearings with an active geometry variation.

2017. Proceedings in applied mathematics and mechanics, 17 (1, SI), 363-364.

doi:10.1002/pamm.201710151 